\title{
6.
}

\section{Über die Eigenschaften der lineären Substitutionen, durch welche eine homogene ganze Function zweiten Grades, welche nur die Quadrate von vier Variabeln enthält, in eine Function von derselben Form transformirt wird.}

(Von dem Herrn O. Hesse, Professor an der Universität zu Königsberg in Pr.)

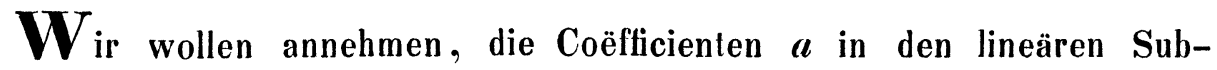
stitutionen

seien so bestimmt, dafs

$$
\left\{\begin{array}{l}
y_{1}=a_{1}^{\prime} x_{1}+a_{1}^{\prime \prime} x_{2}+a_{1}^{(3)} x_{3}+a_{1}^{(4)} x_{4} \\
y_{2}=a_{2}^{\prime} x_{1}+a_{2}^{\prime \prime} x_{2}+a_{2}^{(3)} x_{3}+a_{2}^{(4)} x_{4} \\
y_{3}=a_{3}^{\prime} x_{1}+a_{3}^{\prime \prime} x_{2}+a_{3}^{(3)} x_{3}+a_{3}^{(4)} x_{4} \\
y_{4}=a_{4}^{\prime} x_{1}+a_{4}^{\prime \prime} x_{2}+a_{4}^{(3)} x_{3}+a_{4}^{(4)} x_{4}
\end{array}\right.
$$

$$
b_{1} y_{1}^{2}+b_{2} y_{2}^{2}+b_{3} y_{3}^{2}+b_{4} y_{4}^{2}=a_{1} x_{1}^{2}+a_{2} x_{2}^{2}+a_{3} x_{3}^{2}+a_{4} x_{4}^{2} \text { ist. }
$$

Differentiirt man dieser Annahme gemäfs die letzte Gleichung nach einander nach den unabhängigen Variabeln $x_{1}, x_{2}, x_{3}, x_{4}$, indem man die Gröfsen $y$ als Functionen von den Variabeln $x$ betrachtet, wie sie durch die Substitutionen gegeben sind, so erhält man:

$$
\left\{\begin{array}{l}
a_{1} x_{1}=a_{1}^{\prime} b_{1} y_{1}+a_{2}^{\prime} b_{2} y_{2}+a_{3}^{\prime} b_{3} y_{3}+a_{4}^{\prime} b_{4} y_{4}, \\
a_{2} x_{2}=a_{1}^{\prime \prime} b_{1} y_{1}+a_{2}^{\prime \prime} b_{2} y_{2}+a_{3}^{\prime \prime} b_{3} y_{3}+a_{4}^{\prime \prime} b_{4} y_{4}, \\
a_{3} x_{3}=a_{1}^{(3)} b_{1} y_{1}+a_{2}^{(3)} b_{2} y_{2}+a_{3}^{(3)} b_{3} y_{3}+a_{4}^{(3)} b_{4} y_{4}, \\
a_{4} x_{4}=a_{1}^{(4)} b_{1} y_{1}+a_{2}^{(4)} b_{2} y_{2}+a_{3}^{(4)} b_{3} y_{3}+a_{4}^{(4)} b_{4} y_{4} .
\end{array}\right.
$$

Diese Gleichungen sind als die Auflösungen der Gleichung (1.) nach den Variabeln $x$ zu betrachten und können als solche die Stelle der Gleichungen (1.) vertreten. Wenn man nun $a_{1} x_{1}=Y_{1}, a_{2} x_{2}=Y_{2}, a_{3} x_{3}=Y_{3}, a_{4} x_{4}=Y_{4}$; $b_{1} y_{1}=\boldsymbol{X}_{1}, b_{2} y_{2}=\boldsymbol{X}_{2}, b_{3} y_{3}=\boldsymbol{X}_{3}, b_{4} y_{4}=\boldsymbol{X}_{4}$ setzt, so gehen diese Gleichungen in die Substitutionen

$$
\text { (4.) } \quad\left\{\begin{array}{l}
\boldsymbol{Y}_{1}=a_{1}^{\prime} \boldsymbol{X}_{1}+a_{2}^{\prime} \boldsymbol{X}_{2}+a_{3}^{\prime} \boldsymbol{X}_{3}+a_{4}^{\prime} \boldsymbol{X}_{4}, \\
\boldsymbol{Y}_{2}=a_{2}^{\prime \prime} \boldsymbol{X}_{1}+a_{2}^{\prime \prime} \boldsymbol{X}_{2}+\boldsymbol{a}_{3}^{\prime \prime} \boldsymbol{X}_{3}+\boldsymbol{a}_{4}^{\prime \prime} \boldsymbol{X}_{4} \\
\boldsymbol{Y}_{3}=\boldsymbol{a}_{1}^{(3)} \boldsymbol{X}_{1}+\boldsymbol{a}_{2}^{(3)} \boldsymbol{X}_{2}+\boldsymbol{a}_{3}^{(3)} \boldsymbol{X}_{3}+\boldsymbol{a}_{4}^{(3)} \boldsymbol{X}_{4} \\
\boldsymbol{Y}_{4}=\boldsymbol{a}_{1}^{(4)} \boldsymbol{X}_{1}+\boldsymbol{a}_{2}^{(4)} \boldsymbol{X}_{2}+\boldsymbol{a}_{3}^{(4)} \boldsymbol{X}_{3}+\boldsymbol{a}_{4}^{(4)} \boldsymbol{X}_{4}
\end{array}\right.
$$

Crelle's Journal f. d. Math. Bd. XLV. Heft 2. 
über, in welchen die horizontalen Reihen der Coëfficienten den entsprechenden verticalen Reihen der Coëfficienten in (1.) gleich sind. Die Gleichung (2.) geht über in:

$$
\frac{1}{a_{1}} Y_{1}^{2}+\frac{1}{a_{2}} Y_{2}^{2}+\frac{1}{a_{3}} Y_{3}^{2}+\frac{1}{a_{4}} Y_{4}^{2}=\frac{1}{b_{1}} X_{1}^{2}+\frac{1}{b_{2}} X_{2}^{2}+\frac{1}{b_{3}} X_{3}^{2}+\frac{1}{b_{4}} X_{4}^{2} .
$$

Hierdurch ist, wenn man die Zahlen $1,2,3,4$ durch $k$ bezeichnet, folgender Lehrsatz bewiesen.

\section{Lehrsatz 1 .}

Wenn die Substitutionen $y_{k}=a_{k}^{\prime} x_{1}+a_{k}^{\prime \prime} x_{2}+a_{k}^{(3)} x_{3}+a_{k}^{(4)} x_{4}$ den Ausdruck $b_{1} y_{1}^{2}+b_{2} y_{2}^{2}+b_{3} y_{3}^{2}+b_{4} y_{4}^{2}$ in $a_{1} x_{1}^{2}+a_{2} x_{2}^{2}+a_{3} x_{3}^{2}+a_{4} x_{4}^{2}$ verwandeln, so transformiren die Substitutionen $\boldsymbol{Y}_{k}=a_{1}^{(k)} X_{1}+a_{2}^{(k)} X_{2}+a_{3}^{(k)} X_{3}+a_{4}^{(k)} X_{4}$ den Ausdruck

$$
\frac{1}{a_{1}} Y_{1}^{2}+\frac{1}{a_{2}} Y_{2}^{2}+\frac{1}{a_{3}} Y_{3}^{2}+\frac{1}{a_{4}} Y_{4}^{2} \text { in } \frac{1}{b_{1}} X_{1}^{2}+\frac{1}{b_{2}} X_{2}^{2}+\frac{1}{b_{3}} X_{3}^{2}+\frac{1}{b_{4}} X_{4}^{2} .
$$

Ich führe diesen auf der Hand liegenden Satz, der auch für eine beliebige Zahl von Variabeln gilt, an, um nun aus ihm neue Sätze abzuleiten, welche nur bei vier Variabeln Statt finden.

Da die horizontalen Reihen der Coëfficienten in den Gleichungen (4.) den entsprechenden variabeln Reihen in den.Gleichungen (1.) gleich sind, so wird auch bei den Auflösungen der beiden Systeme Gleichungen das Nemliche Statt finden. Es ergeben sich daher folgende Auflösungen der Gleichungen (4.):

$$
\left\{\begin{array}{l}
\frac{1}{b_{1}} X_{1}=a_{1}^{\prime} \frac{1}{a_{1}} Y_{1}+a_{1}^{\prime \prime} \frac{1}{a_{2}} Y_{2}+a_{1}^{(3)} \frac{1}{a_{3}} Y_{3}+a_{1}^{(4)} \frac{1}{a_{4}} Y_{4}, \\
\frac{1}{b_{2}} X_{2}=a_{2}^{\prime} \frac{1}{a_{1}} Y_{1}+a_{2}^{\prime \prime} \frac{1}{a_{2}} Y_{2}+a_{2}^{(3)} \frac{1}{a_{3}} Y_{3}+a_{2}^{(4)} \frac{1}{a_{4}} Y_{4}, \\
\frac{1}{b_{3}} X_{3}=a_{3}^{\prime} \frac{1}{a_{1}} Y_{1}+a_{3}^{\prime \prime} \frac{1}{a_{2}} Y_{2}+a_{3}^{(3)} \frac{1}{a_{3}} Y_{3}+a_{3}^{(4)} \frac{1}{a_{4}} Y_{4}, \\
\frac{1}{b_{4}} X_{4}=a_{4}^{\prime} \frac{1}{a_{1}} Y_{1}+a_{4}^{\prime \prime} \frac{1}{a_{2}} Y_{2}+a_{4}^{(3)} \frac{1}{a_{3}} Y_{3}+a_{4}^{(4)} \frac{1}{a_{4}} Y_{4}
\end{array}\right.
$$

welche Gleichungen man auch aus (5.) durch Differentiation, eben so wie die Gleichungen (3.) und (2.), ableiten kann. Setzt man die Werthe von $y_{1}, y_{2}, \ldots$ aus (1.) in (3.), so ergeben sich, durch Gleichstellung der Coëfficienten gleicher Variabeln auf beiden Seiten der Gleichungen, folgende Ausdrücke:

$$
\left\{\begin{array}{l}
a_{k}=b_{1} a_{1}^{k} a_{1}^{k}+b_{2} a_{2}^{k} a_{2}^{k}+b_{3} a_{3}^{k} a_{3}^{k}+b_{4} a_{4}^{k} a_{4}^{k}, \\
0=b_{1} a_{1}^{k} a_{1}^{\lambda}+b_{2} a_{2}^{k} a_{2}^{\lambda}+b_{3} a_{3}^{k} a_{3}^{\alpha}+b_{4} a_{4}^{k} a_{4}^{\lambda},
\end{array}\right.
$$


6. Hesse, über Verwandlungen der Functionen zweiten Grades.

und auf gleiche Weise aus (4. und 6.)

$$
\left\{\begin{array}{l}
\frac{1}{b_{k}}=\frac{1}{a_{1}} a_{k}^{\prime} a_{k}^{\prime}+\frac{1}{a_{2}} a_{k}^{\prime \prime} a_{k}^{\prime \prime}+\frac{1}{a_{3}} a_{k}^{(3)} a_{k}^{(3)}+\frac{1}{a_{4}} a_{k}^{(4)} a_{k}^{(4)}, \\
0=\frac{1}{a_{1}} a_{k}^{\prime} a_{\lambda}^{\prime}+\frac{1}{a_{2}} a_{k}^{\prime \prime} a_{\lambda}^{\prime \prime}+\frac{1}{a_{3}} a_{k}^{(3)} a_{\lambda}^{(3)}+\frac{1}{a_{4}} a_{k}^{(4)} a_{\lambda}^{(4)} .
\end{array}\right.
$$

Wir wollen nun untersuchen, was die Substitutionen

$$
\begin{aligned}
\text { (9.) } y_{k} & =\frac{1}{a_{k}^{\prime}} x_{1}+\frac{1}{a_{k}^{\prime \prime}} x_{2}+\frac{1}{a_{k}^{(3)}} x_{3}+\frac{1}{a_{k}^{(+)}} x_{4}, \\
\text { (10.) } \quad Y_{k} & =\frac{1}{a_{1}^{(k)}} X_{1}+\frac{1}{a_{2}^{(k)}} X_{2}+\frac{1}{a_{3}^{(k)}} X_{3}+\frac{1}{a_{4}^{(k)}} X_{4}
\end{aligned}
$$

geben.

Zu diesem Ende werde bemerkt, dafs von den Bedingungsgleichungen $0=b_{1} a_{1}^{k} a_{1}^{\lambda}+b_{2} a_{2}^{k} a_{2}^{\lambda}+b_{3} a_{3}^{k} a_{3}^{\lambda}+b_{4} a_{4}^{k} a_{4}^{\lambda}$, welche erfüllt werden müssen, wenn in dem durch die Substitutionen (1.) zu transformirenden Ausdrucke $b_{1} y_{1}^{2}+b_{2} y_{2}^{2}+b_{3} y_{3}^{2}+b_{4} y_{4}^{2}$ die Producte der Variabeln $x_{1}, x_{2}, \ldots$ verschwinden sollen, die eine in die andere übergeht, wenn man $\frac{1}{a_{\mu}^{k}}$ und $\frac{1}{a_{\mu}^{\lambda}}$ statt $a_{\mu}^{k}$ und $a_{\mu}^{\lambda}$ und zugleich $b_{k} a_{k}^{\prime} a_{k}^{\prime \prime} a_{k}^{(3)} a_{k}^{(4)}$ statt $b_{k}$ setzt. Von den Bedingungsgleichungen $0=\frac{1}{a_{1}} a_{k}^{\prime} a_{\lambda}^{\prime}+\frac{1}{a_{2}} a_{k}^{\prime \prime} a_{\lambda}^{\prime \prime}+\frac{1}{a_{3}} a_{k}^{(3)} a_{\lambda}^{(3)}+\frac{1}{a_{4}} a_{k}^{(4)} a_{\lambda}^{(4)}$, welche erfüllt werden müssen, wenn in dem durch (4.) zu transformirenden Ausdrucke $\frac{1}{a_{1}} Y_{1}^{2}+\frac{1}{a_{2}} Y_{2}^{2}$ $+\frac{1}{a_{3}} Y_{3}^{2}+\frac{1}{a_{4}} Y_{4}^{2}$ die Producte der Variabeln $X_{1}, X_{2}, \ldots$ wegfallen, geht ferner die eine in die andere über, wenn man $\frac{1}{a_{\mu}^{k}}$ und $\frac{1}{a_{\mu}^{\lambda}}$ statt $a_{\mu}^{k}$ und $a_{\mu}^{\lambda}$ und zugleich $\frac{1}{a_{k}} a_{1}^{k} a_{2}^{k} a_{3}^{k} a_{4}^{k}$ statt $\frac{1}{a^{k}}$ setzt.

Hieraus folgt, wenn man der Kürze wegen

$$
\text { (11.) } \quad \boldsymbol{a}_{k}^{\prime} \boldsymbol{a}_{k}^{\prime \prime} \boldsymbol{a}_{k}^{(3)} \boldsymbol{a}_{k}^{(4)}=\boldsymbol{A}_{k}, \quad \boldsymbol{a}_{1}^{k} \boldsymbol{a}_{2}^{k} \boldsymbol{a}_{3}^{k} \boldsymbol{a}_{4}^{k}=\boldsymbol{A}^{k}
$$

setzt, dafs durch die Substitutionen (9. und 10.) die Ausdrücke

(12.) $b_{1} A_{1} y_{1}^{2}+b_{2} a_{2} y_{2}^{2}+b_{3} a_{3} y_{3}^{2}+b_{4} a_{4} y_{4}^{2}$,

$$
\frac{A^{\prime}}{a_{1}} \boldsymbol{Y}_{1}^{2}+\frac{A^{\prime \prime}}{a_{2}} \boldsymbol{Y}_{2}^{2}+\frac{A^{3}}{a_{3}} \boldsymbol{Y}_{3}^{2}+\frac{A^{4}}{a_{4}} \boldsymbol{Y}_{4}^{2}
$$

in solche transformirt werden, die nur die Quadrate der neuen Variabeln enthalten.

Nimmt man an, der Ausdruck (12.) gehe durch die Substitutionen (9.) in folgenden

$$
\text { (14.) } \quad c_{1} A_{1} x_{1}^{2}+c_{2} A_{2} x_{2}^{2}+c_{3} A_{3} x_{3}^{2}+c_{4} A_{4} x_{4}^{2}
$$


über, so mufs nach dem aufgestellten Lehrsatze der Ausdruck

$$
\frac{1}{c_{1} A_{1}} Y_{1}^{2}+\frac{1}{c_{2} A_{2}} Y_{2}^{2}+\frac{1}{c_{3} A_{3}} Y_{3}^{2}+\frac{1}{c_{4} A_{4}} Y_{4}^{2}
$$

durch die Substitutionen (10.) in

$$
\frac{1}{b_{1} A_{1}} X_{1}^{2}+\frac{1}{b_{2} A_{2}} X_{2}^{2}+\frac{1}{b_{3} A_{3}} X_{3}^{2}+\frac{1}{b_{4} A_{4}} X_{4}^{2}
$$

übergehen.

Wir haben nun zwei verschiedene Functionen der Variabeln $\boldsymbol{Y}_{1}, \boldsymbol{Y}_{2}, \ldots$, welche nur die Quadrate der Variabeln enthalten, nämlich (13. und 15.), welche beide durch die Substitutionen (16.) in solche Funclionen der Variabeln $\boldsymbol{X}_{1}, \boldsymbol{X}_{2}, \ldots$ übergehen, die nur die Quadrate dieser letzteren Variaḅeln enthalten. Die Zahl der Bedingungsgleichungén, welche zu erfüllen sind, wenn dieses zutreffen soll, ist für jede dieser Functionen sechs. Nimmt man nun die Coëfficienten in den Substitutionen als gegeben an, betrachtet dagegen die 4 Coëfficienten in den Functionen (13. und 15.) in den erwähnten Bedingungsgleichungen :als die gesuchten Gröfsen, so hat man dieselben lineären Gleichungen zur Bestimmung der Werthe der 4 Coëfficienten für die eine, wie für die andere Function. Hieraus ist ersichtlich, dafs die entsprechenden Coëfficienten in den beiden Functionen nur durch einen Factor $\boldsymbol{M}$ von einander verschieden sein können. Es ist daher

$c_{1} A_{1}=M \cdot \frac{a_{1}}{A^{\prime}}, \quad c_{2} A_{2}=M \cdot \frac{a_{2}}{A^{\prime \prime}}, \quad c_{3} A_{3}=M \cdot \frac{a_{3}}{A^{(3)}}, \quad c_{4} A_{4}=M \cdot \frac{a_{4}}{A^{(4)}} \cdot$

Setzt man diese Werthe von $c_{1} A_{1}, c_{2} A_{2}, \ldots$ in (14.), so erhält man den Ausdruck

$$
\boldsymbol{M}\left(\frac{a_{1}}{A^{\prime}} x_{1}^{2}+\frac{a_{2}}{A^{\prime \prime}} x_{2}^{2}+\frac{a_{3}}{A^{(3)}} x_{3}^{2}+\frac{a_{4}}{A^{(4)}} x_{4}^{2}\right),
$$

in welchen der Ausdruck (12.) durch die Substitutionen (9.) übergeht.

Der Ausdruck (13.) geht demnach, mit Rücksicht auf den oben angegebenen Lehrsatz, durch die Substitutionen (10.) in

$$
\text { (18.) } \quad M\left(\frac{1}{b_{1} A_{1}} X_{1}^{2}+\frac{1}{b_{2} A_{2}} X_{2}^{2}+\frac{1}{b_{3} A_{3}} X_{3}^{2}+\frac{1}{b_{4} A_{4}} X_{4}^{2}\right)
$$

über. Diese Bemerkungen vereinigen sich in dem folgenden Lehrsatze.

Lehrsatz 2.

Wenn die Substitutionen $y_{k}=a_{k}^{\prime} x_{1}+a_{k}^{\prime \prime} x_{2}+a_{k}^{(3)} x_{3}+a_{k}^{(4)} x_{4}$ den Ausdruck $b_{1} y_{1}^{2}+b_{2} y_{2}^{2}+b_{3} y_{3}^{2}+b_{4} y_{4}^{2}$ in $a_{1} x_{1}^{2}+a_{2} x_{2}^{2}+a_{3} x_{3}^{2}+a_{4} x_{4}^{2}$ verwandeln, 
6. Hesse, ̈̈ber Verwandlungen der Functionen zweiten Grades.

so transformiren die Substitutionen

$$
y_{k}=\frac{1}{a_{k}^{\prime}} x_{1}+\frac{1}{a_{k}^{\prime \prime}} x_{2}+\frac{1}{a_{k}^{(3)}} x_{3}+\frac{1}{a_{k}^{(4)}} x_{4}
$$

den Ausdruck $b_{1} A_{1} y_{1}^{2}+b_{2} A_{2} y_{2}^{2}+b_{3} A_{3} y_{3}^{2}+b_{4} A_{4} y_{4}^{2}$ in

$$
\boldsymbol{M}\left(\frac{a_{1}}{A^{\prime}} x_{1}^{2}+\frac{a_{2}}{A^{\prime \prime}} x_{2}^{2}+\frac{a_{3}}{A^{(3)}} x_{3}^{2}+\frac{a_{4}}{A^{(4)}} x_{4}^{2}\right),
$$

und die Substitutionen $Y_{k}=\frac{1}{a_{1}^{k}} \dot{X}_{1}+\frac{1}{a_{2}^{k}} X_{2}+\frac{1}{a_{3}^{k}} X_{3}+\frac{1}{a_{4}^{k}} X_{4}$ transformiren den Ausdruck $\frac{A^{\prime}}{a_{1}} Y_{1}^{2}+\frac{A^{\prime \prime}}{a_{2}} Y_{2}^{2}+\frac{A^{(3)}}{a_{3}} Y_{3}^{2}+\frac{A^{(4)}}{a_{4}} Y_{4}^{2}$ in

$$
\boldsymbol{M}\left(\frac{1}{b_{1} A_{1}} X_{1}^{2}+\frac{1}{b_{2} A_{2}} X_{2}^{2}+\frac{1}{b_{3} A_{3}} X_{3}^{2}+\frac{1}{b_{4} A_{4}} X_{4}^{2}\right) \text {. }
$$

Ich füge noch hinzu, dafs die Werthe der Gröfsen $\boldsymbol{A}$ in (11.) gegeben sind und dafs der Factor $\boldsymbol{M}$ durch die Gleichung

$$
\boldsymbol{M} \frac{a_{k}}{A^{k}}=\frac{b_{1} A_{1}}{a_{1}^{k} a_{1}^{k}}+\frac{b_{2} A_{2}}{a_{2}^{k} a_{2}^{k}}+\frac{b_{3} A_{3}}{a_{3}^{k} a_{3}^{k}}+\frac{b_{4} A_{4}}{a_{4}^{k} a_{4}^{k}}
$$

bestimmt wird, welche man durch Vergleichung von (12. und 17.) erhält. Andere merkwürdige Relationen, die sich aus dem angeführten Lehrsatze ohne Schwierigkeit ableiten lassen, übergehe ich, weil sie Formeln geben würden, die in der folgenden Untersuchung keine Anwendung finden.

Es sei

(20.) $\quad \boldsymbol{F}=b_{12} y_{1} y_{2}+b_{13} y_{1} y_{3}+b_{14} y_{1} y_{4}+b_{23} y_{2} y_{3}+b_{24} y_{2} y_{4}+b_{34} y_{3} y_{4}$ eine homogene Function zweiten Grades, in welcher die Quadrate der Variabeln fehlen, und welche die Eigenschaft hat, dafs sie durch die Substitutionen (1.), vermöge welcher die identische Gleichung (2.) Statt findet, in eine Function der Variabeln $x_{1}, x_{2}, \ldots$ von derselben Form übergeht, nämlich in

(21.) $\quad F=a_{12} x_{1} x_{2}+a_{13} x_{1} x_{3}+a_{14} x_{1} x_{4}+a_{23} x_{2} x_{3}+a_{24} x_{2} x_{4}+a_{34} x_{3} x_{4}$.

In dieser Voraussetzung hat man

$$
\begin{aligned}
& \left\{\begin{aligned}
0= & \boldsymbol{b}_{12} a_{1}^{k} a_{2}^{k}+b_{13} a_{1}^{k} a_{3}^{k}+b_{14} a_{1}^{k} a_{4}^{k}+b_{23} a_{2}^{k} a_{3}^{k}+b_{24} a_{2}^{k} a_{4}^{k}+b_{34} a_{3}^{k} a_{4}^{k} \\
a_{k \lambda}= & b_{12}\left(a_{1}^{k} a_{2}^{\lambda}+a_{1}^{\lambda} a_{2}^{k}\right)+b_{13}\left(a_{1}^{k} a_{3}^{\lambda}+a_{1}^{\lambda} a_{3}^{k}\right)+b_{14}\left(a_{1}^{k} a_{4}^{\lambda}+a_{1}^{\lambda} a_{4}^{k}\right) \\
& +b_{23}\left(a_{2}^{k} a_{3}^{\lambda}+a_{2}^{\lambda} a_{3}^{k}\right)+b_{24}\left(a_{2}^{k} a_{4}^{\lambda}+a_{2}^{\lambda} a_{4}^{k}\right)+b_{34}\left(a_{3}^{k} a_{4}^{\lambda}+a_{3}^{\lambda} a_{4}^{k}\right)
\end{aligned}\right. \\
& \left\{\begin{aligned}
0= & \frac{a_{12}}{a_{1} a_{2}} a_{k}^{\prime} a_{k}^{\prime \prime}+\frac{a_{13}}{a_{1} a_{3}} a_{k}^{\prime} a_{k}^{3}+\frac{a_{14}}{a_{1} a_{4}} a_{k}^{\prime} a_{k}^{4}+\frac{a_{23}}{a_{2} a_{3}} a_{k}^{\prime \prime} a_{k}^{3}+\frac{a_{24}}{a_{2} a_{4}} a_{k}^{\prime \prime} a_{k}^{4}+\frac{a_{34}}{a_{3} a_{4}} a_{k}^{3} a_{k}^{4} \\
b_{k \lambda}= & \frac{a_{12}}{a_{1} a_{2}}\left(a_{k}^{\prime} a_{\lambda}^{\prime \prime}+a_{k}^{\prime \prime} a_{\lambda}^{\prime}\right)+\frac{a_{13}}{a_{1} a_{3}}\left(a_{k}^{\prime} a_{\lambda}^{3}+a_{k}^{3} a_{\lambda}^{\prime}\right)+\frac{a_{14}}{a_{1} a_{4}}\left(a_{k}^{\prime} a_{\lambda}^{4}+a_{k}^{4} a_{\lambda}^{\prime}\right) \\
+ & \frac{a_{23}}{a_{2} a_{3}}\left(a_{k}^{\prime \prime} a_{\lambda}^{3}+a_{k}^{3} a_{\lambda}^{\prime \prime}\right)+\frac{a_{24}}{a_{2} a_{4}}\left(a_{k}^{\prime \prime} a_{\lambda}^{4}+a_{k}^{4} a_{\lambda}^{\prime \prime}\right)+\frac{a_{34}}{a_{3} a_{4}}\left(a_{k}^{3} a_{\lambda}^{4}+a_{k}^{4} a_{\lambda}^{3}\right)
\end{aligned}\right.
\end{aligned}
$$


Dividirt man die Function $\boldsymbol{F}$ durch das Product $y_{1} y_{2} y_{3} y_{4}$ und selzt hierauf $y_{1}, y_{2}, y_{3}, y_{4}$ statt $\frac{1}{y_{1}}, \frac{1}{y_{2}}, \frac{1}{y_{3}}, \frac{1}{y_{4}}$, so erhält man die neue Function

(24.) $\Phi=b_{34} y_{1} y_{2}+b_{24} y_{1} y_{3}+b_{23} y_{1} y_{4}+b_{14} y_{2} y_{3}+b_{13} y_{2} y_{4}+b_{12} y_{3} y_{4}$.

Wir wollen nun untersuchen, in welche Function diese Function übergeht, wenn man in derselben die Substitutionen (9.) macht.

Es ist leicht zu sehen, dafs in der durch (9.) transformirten Function $\Phi$ die Quadrate der Variabeln $x_{1}, x_{2}, \ldots$ vermöge der Gleichungen (21.) wegfallen. Die transformirte Function $\Phi$ hat mithin die nemliche Form wie die Function $\Phi$ selber, nämlich :

(25.) $\Phi=A_{34} x_{1} x_{2}+A_{24} x_{1} x_{3}+A_{23} x_{1} x_{4}+A_{14} x_{2} x_{3}+A_{13} x_{2} x_{4}+A_{12} x_{3} x_{4}$, und die Coëfficienten $\boldsymbol{A}$ erhalten die Werthe

$$
\left\{\begin{aligned}
A_{12}= & b_{34}\left(\frac{1}{a_{1}^{3} a_{2}^{4}}+\frac{1}{a_{1}^{4} a_{2}^{3}}\right)+b_{24}\left(\frac{1}{a_{1}^{3} a_{3}^{4}}+\frac{1}{a_{1}^{4} a_{3}^{3}}\right)+b_{23}\left(\frac{1}{a_{1}^{3} a_{4}^{4}}+\frac{1}{a_{1}^{4} a_{4}^{3}}\right) \\
& +b_{14}\left(\frac{1}{a_{2}^{3} a_{2}^{4}}+\frac{1}{a_{2}^{4} a_{3}^{3}}\right)+b_{13}\left(\frac{1}{a_{2}^{3} a_{4}^{4}}+\frac{1}{a_{2}^{4} a_{4}^{3}}\right)+b_{12}\left(\frac{1}{a_{3}^{3} a_{4}^{4}}+\frac{1}{a_{3}^{4} a_{4}^{3}}\right) \\
A_{13}= & b_{34}\left(\frac{1}{a_{1}^{\prime \prime} a_{2}^{4}}+\frac{1}{a_{1}^{4} a_{2}^{\prime \prime}}\right)+b_{24}\left(\frac{1}{a_{1}^{\prime \prime} a_{3}^{4}}+\frac{1}{a_{1}^{4} a_{3}^{\prime \prime}}\right)+\cdots
\end{aligned}\right.
$$

Diese Coëfficienten $\boldsymbol{A}$ lassen sich durch die Coëfficienten $\boldsymbol{a}$ der transformirten Function $\boldsymbol{F}$ sehr einfach ausdrücken. Um dazu zu gelangen, dienen folgende Betrachtungen.

Die in (20.) angegebene Function $\boldsymbol{F}$, welche durch die Snbstitutionen (1.) in die in (21.) angegebene Function ũbergeht, läfst sich als eine Function der Variabeln $y_{1}, y_{2}, \ldots$ oder als eine Function der Variabeln $x_{1}, x_{2}, \ldots$ betrachten, indem die ersteren Variabeln als Functionen der zweiten durch die Substitutionen (1.) gegeben sind, oder die zweiten Variabeln als Functionen der ersteren durch die Gleichungen (3.). Differentiirt man die Function $\boldsymbol{F}$ unter der letzteren Annahme nach den unabhängigen Variabeln $y_{1}, y_{2}, \ldots$, so ergiebt sich

$$
\begin{aligned}
& \frac{1}{b_{1}} \frac{\partial \boldsymbol{F}}{\partial y_{1}}=\frac{1}{a_{1}} \frac{\partial \boldsymbol{F}}{\partial x_{1}} \cdot a_{1}^{\prime}+\frac{1}{a_{2}} \frac{\partial \boldsymbol{F}}{\partial x_{2}} \cdot a_{1}^{\prime \prime}+\frac{1}{a_{3}} \frac{\partial F}{\partial x_{3}} \cdot a_{1}^{3}+\frac{1}{a_{4}} \frac{\partial F}{\partial x_{4}} \cdot a_{1}^{4} \\
& \frac{1}{b_{2}} \frac{\partial F}{\partial y_{2}}=\frac{1}{a_{1}} \frac{\partial F}{\partial x_{1}} \cdot a_{2}^{\prime}+\frac{1}{a_{2}} \frac{\partial F}{\partial x_{2}} \cdot a_{2}^{\prime \prime}+\frac{1}{a_{3}} \frac{\partial F}{\partial x_{3}} \cdot a_{2}^{3}+\frac{1}{a_{4}} \frac{\partial F}{\partial x_{4}} \cdot a_{2}^{4} \\
& \frac{1}{b_{3}} \frac{\partial F}{\partial y_{3}}=\frac{1}{a_{1}} \frac{\partial F}{\partial x_{1}} \cdot a_{3}^{\prime}+\frac{1}{a_{2}} \frac{\partial F}{\partial x_{2}} \cdot a_{3}^{\prime \prime}+\frac{1}{a_{3}} \frac{\partial F}{\partial x_{3}} \cdot a_{3}^{3}+\frac{1}{a_{4}} \frac{\partial F}{\partial x_{4}} \cdot a_{3}^{4}, \\
& \frac{1}{b_{4}} \frac{\partial F}{\partial y_{4}}=\frac{1}{a_{1}} \frac{\partial F}{\partial x_{1}} \cdot a_{4}^{\prime}+\frac{1}{a_{2}} \frac{\partial F}{\partial x_{2}} \cdot a_{4}^{\prime \prime}+\frac{1}{a_{3}} \frac{\partial F}{\partial x_{3}} \cdot a_{4}^{3}+\frac{1}{a_{4}} \frac{\partial F}{\partial x_{4}} \cdot a_{4}^{4}
\end{aligned}
$$


Setzt man in diesen Gleichungen für die partiellen Differentialquotienten ihre Werthe, und $y_{1}=a_{1}^{4}, y_{2}=a_{2}^{4}, y_{3}=a_{3}^{4}, y_{4}=a_{4}^{4}$, zugleich mit den entsprechenden Werthen der Variabeln, $x_{1}=x_{2}=x_{3}=0, x_{4}=1$, so erhält man

$$
\begin{aligned}
& \frac{1}{b_{1}}\left(b_{12} a_{2}^{4}+b_{13} a_{3}^{4}+b_{14} a_{4}^{4}\right)=\frac{a_{41} a_{1}^{\prime}}{a_{1}}+\frac{a_{42} a_{1}^{\prime \prime}}{a_{2}}+\frac{a_{43} a_{1}^{3}}{a_{3}}, \\
& \frac{1}{b_{2}}\left(b_{21} a_{1}^{4}+b_{23} a_{3}^{4}+b_{24} a_{4}^{4}\right)=\frac{a_{41} a_{2}^{\prime}}{a_{1}}+\frac{a_{42} a_{2}^{\prime \prime}}{a_{2}}+\frac{a_{43} a_{2}^{3}}{a_{3}}, \\
& \frac{1}{b_{3}}\left(b_{31} a_{1}^{4}+b_{33} a_{2}^{4}+b_{34} a_{4}^{4}\right)=\frac{a_{41} a_{3}^{\prime}}{a_{1}}+\frac{a_{42} a_{3}^{\prime \prime}}{a_{2}}+\frac{a_{43} a_{3}^{3}}{a_{3}}, \\
& \frac{1}{b_{4}}\left(b_{41} a_{1}^{4}+b_{42} a_{2}^{4}+b_{43} a_{3}^{4}\right)=\frac{a_{41} a_{4}^{\prime}}{a_{1}}+\frac{a_{42} a_{4}^{\prime \prime}}{a_{2}}+\frac{a_{43} a_{4}^{3}}{a_{3}} .
\end{aligned}
$$

Multiplicirt man diese Gleichungen der Reihe nach mit $a_{1}^{4}, a_{2}^{4}, a_{3}^{4}, a_{4}^{4}$, so lassen sich die Theile derselben links durch Hülfe der Gleichungen (22.) und die Theile rechts mit Hülfe der Gleichungen (23.) auf die Weise umformen, dafs

$$
\begin{aligned}
& \frac{1}{b_{1}}\left(b_{34} a_{3}^{4} a_{4}^{4}+b_{24} a_{2}^{4} a_{4}^{4}+b_{23} a_{2}^{4} a_{3}^{4}\right)=a_{4}\left(\frac{a_{12} a_{1}^{\prime} a_{1}^{\prime \prime}}{a_{1} a_{2}}+\frac{a_{13} a_{1}^{\prime} a_{1}^{3}}{a_{1} a_{3}}+\frac{a_{23} a_{1}^{\prime \prime} a_{1}^{3}}{a_{2} a_{3}}\right), \\
& \frac{1}{b_{2}}\left(b_{43} a_{3}^{4} a_{4}^{4}+b_{14} a_{1}^{4} a_{4}^{4}+b_{13} a_{1}^{4} a_{3}^{4}\right)=a_{4}\left(\frac{a_{12} a_{2}^{\prime} a_{2}^{\prime \prime}}{a_{1} a_{2}}+\frac{a_{13} a_{2}^{\prime} a_{2}^{3}}{a_{1} a_{3}}+\frac{a_{23} a_{2}^{\prime \prime} a_{2}^{3}}{a_{2} a_{3}}\right), \\
& \frac{1}{b_{3}}\left(b_{24} a_{2}^{4} a_{4}^{4}+b_{14} a_{1}^{4} a_{4}^{4}+b_{12} a_{1}^{4} a_{2}^{4}\right)=a_{4}\left(\frac{a_{12} a_{3}^{\prime} a_{3}^{\prime \prime}}{a_{1} a_{2}}+\frac{a_{13} a_{3}^{\prime} a_{3}^{3}}{a_{1} a_{3}}+\frac{a_{23} a_{3}^{\prime \prime} a_{3}^{3}}{a_{2} a_{3}}\right), \\
& \frac{1}{b_{4}}\left(b_{23} a_{2}^{4} a_{3}^{4}+b_{13} a_{1}^{4} a_{3}^{4}+b_{12} a_{1}^{4} a_{2}^{4}\right)=a_{4}\left(\frac{a_{12} a_{4}^{\prime} a_{4}^{\prime \prime}}{a_{1} a_{2}}+\frac{a_{13} a_{4}^{\prime} a_{4}^{3}}{a_{1} a_{3}}+\frac{a_{23} a_{4}^{\prime \prime} a_{4}^{3}}{a_{2} a_{3}}\right) .
\end{aligned}
$$

Multiplicirt man diese Gleichungen der Reihe nach mit

$$
\frac{b_{1}}{a_{2}^{4} a_{3}^{4} a_{4}^{4}}=\frac{b_{1} a_{1}^{4}}{A^{4}}, \quad \frac{b_{2}}{a_{1}^{4} a_{3}^{4} a_{4}^{4}}=\frac{b_{2} a_{2}^{4}}{A^{4}}, \quad \frac{b_{3}}{a_{1}^{4} a_{2}^{4} a_{4}^{4}}=\frac{b_{3} a_{3}^{4}}{A^{4}}, \quad \frac{b_{4}}{a_{1}^{4} a_{2}^{4} a_{3}^{4}}=\frac{b_{4} a_{4}^{4}}{A^{4}}
$$

so erhält man

$$
\begin{aligned}
& b_{34} \frac{1}{a_{2}^{4}}+b_{24} \frac{1}{a_{3}^{4}}+b_{23} \frac{1}{a_{4}^{4}}=\frac{b_{1} a_{4} a_{1}^{4}}{A^{4}}\left(\frac{a_{12} a_{1}^{\prime} a_{1}^{\prime \prime}}{a_{1} a_{2}}+\frac{a_{13} a_{1}^{\prime} a_{1}^{3}}{a_{1} a_{3}}+\frac{a_{23} a_{1}^{\prime \prime} a_{1}^{3}}{a_{2} a_{3}}\right), \\
& b_{34} \frac{1}{a_{1}^{4}}+b_{14} \frac{1}{a_{3}^{4}}+b_{13} \frac{1}{a_{4}^{4}}=\frac{b_{2} a_{4} a_{2}^{4}}{A^{4}}\left(\frac{a_{12} a_{2}^{\prime} a_{2}^{\prime \prime}}{a_{1} a_{2}}+\frac{a_{13} a_{2}^{\prime} a_{2}^{3}}{a_{1} a_{3}}+\frac{a_{23} a_{2}^{\prime \prime} a_{2}^{3}}{a_{2} a_{3}}\right), \\
& b_{23} \frac{1}{a_{1}^{4}}+b_{14} \frac{1}{a_{2}^{4}}+b_{12} \frac{1}{a_{4}^{4}}=\frac{b_{3} a_{4} a_{3}^{4}}{A^{4}}\left(\frac{a_{12} a_{3}^{\prime} a_{3}^{\prime \prime}}{a_{1} a_{2}}+\frac{a_{13} a_{3}^{\prime} a_{3}^{3}}{a_{1} a_{3}}+\frac{a_{23} a_{3}^{\prime \prime} a_{3}^{3}}{a_{2} a_{3}}\right), \\
& b_{23} \frac{1}{a_{1}^{4}}+b_{13} \frac{1}{a_{2}^{4}}+b_{12} \frac{1}{a_{3}^{4}}=\frac{b_{4} a_{4} a_{4}^{4}}{A^{4}}\left(\frac{a_{12} a_{4}^{\prime} a_{4}^{\prime \prime}}{a_{1} a_{2}}+\frac{a_{13} a_{4}^{\prime} a_{4}^{3}}{a_{1} a_{3}}+\frac{a_{23} a_{4}^{\prime \prime} a_{4}^{3}}{a_{2} a_{3}}\right) .
\end{aligned}
$$

Multiplicirt man endlich diese Gleichungen der Reihe nach mit $\frac{1}{a_{1}^{3}}, \frac{1}{a_{2}^{3}}$, $\frac{1}{a_{3}^{3}}, \frac{1}{a_{4}^{3}}$ und addirt die Producte, so giebt die Summe der Glieder links gerade 
100 6. Hesse, über Verwandlungen der Functionen zweiten Grades.

den Ausdruck, welcher vorhin mit $\boldsymbol{A}_{12}$ bezeichnet wurde, während rechts die mit $a_{13}$ und $a_{23}$ multiplicirten Glieder wegen der Gleichungen (7.) wegfallen, so dafs sich

$$
A_{12}=\frac{a_{12} \cdot a_{4}}{a_{1} a_{2} A^{4}}\left\{\frac{b_{1} A_{1}}{a_{1}^{3} a_{1}^{3}}+\frac{b_{2} A_{2}}{a_{2}^{3} a_{2}^{3}}+\frac{b_{3} A_{3}}{a_{3}^{3} a_{3}^{3}}+\frac{b_{4} A_{4}}{a_{4}^{3} a_{4}^{3}}\right\}
$$

und nach (19.)

$$
\left\{\begin{array}{l}
A_{12}=a_{12} \cdot \frac{a_{3} a_{3}}{A^{3}} \cdot \frac{a_{4} a_{4}}{A^{4}} \cdot \frac{M}{a_{1} a_{2} a_{3} a_{4}} \text { und eben so } \\
A_{13}=a_{13} \cdot \frac{a_{2} a_{2}}{A^{\prime \prime}} \cdot \frac{a_{4} a_{4}}{A^{4}} \cdot \frac{M}{a_{1} a_{2} a_{3} a_{4}} \\
. . \quad . \quad .
\end{array}\right.
$$

findet. Hierdurch ist folgender Lehrsatz bewiesen.

\section{Lehrsatz 3 .}

Wenn die Substitutionen $y_{k}=a_{k}^{\prime} x_{1}+a_{k}^{\prime \prime} x_{2}+a_{k}^{3} x_{3}+a_{k}^{4} x_{4}$ den Ausdruck $b_{1} y_{1}^{2}+b_{2} y_{2}^{2}+b_{3} y_{3}^{2}+b_{4} y_{4}^{2}$ in $a_{1} x_{1}^{2}+a_{2} x_{2}^{2}+a_{3} x_{3}^{2}+a_{4} x_{4}^{2}$ und überdies dieselben Substitutionen den Ausdruck $b_{12} y_{1} y_{2}+b_{13} y_{1} y_{3}+b_{14} y_{1} y_{4}$ $b_{23} y_{2} y_{3}+b_{24} y_{2} y_{4}+b_{34} y_{3} y_{4}$ in $a_{12} x_{1} x_{2}+a_{13} x_{1} x_{3}+a_{14} x_{1} x_{4}+a_{23} x_{2} x_{3}$ $+a_{24} x_{2} x_{4}+a_{34} x_{3} x_{4}$ verwandeln, so transformiren die Substitutionen $y_{k}=\frac{1}{a_{k}^{\prime}} x_{1}+\frac{1}{a_{k}^{\prime \prime}} x_{2}+\frac{1}{a_{k}^{3}} x_{3}+\frac{1}{a_{k}^{4}} x_{4}$ den Ausdruck $b_{34} y_{1} y_{2}+b_{24} y_{1} y_{3}+b_{23} y_{1} y_{4}$ $b_{14} y_{2} y_{3}+b_{13} y_{2} y_{4}+b_{12} y_{3} y_{4}$ in

$$
\frac{M}{a_{1} a_{2} a_{3} a_{4}}\left\{a_{34} z_{1} z_{2}+a_{24} z_{1} z_{3}+a_{23} z_{2} z_{3}+a_{14} z_{2} z_{3}+a_{13} z_{2} z_{4}+a_{12} z_{3} z_{4}\right\},
$$

wo $z_{1}=\frac{a_{1} a_{1} x_{1}}{A^{\prime}}, \quad z_{2}=\frac{a_{2} a_{2} x_{2}}{A^{\prime \prime}}, \quad z_{3}=\frac{a_{3} a_{3} x_{3}}{A^{3}}, \quad z_{4}=\frac{a_{4} a_{4} x_{4}}{A^{4}}$ und $\boldsymbol{A}^{k}=\boldsymbol{a}_{1}^{k} \boldsymbol{a}_{2}^{k} \boldsymbol{a}_{3}^{k} \boldsymbol{a}_{4}^{k}$ ist.

Die beiden ersten Lehrsälze lassen sich geometrisch wie folgt deuten:

Es seien 8 Puncte im Raume gegeben, in welchen sich drei Oberflächen zweiter Ordnung schneiden. Betrachtet man irgend 4 von diesen Puncten als die Ecken eines Tetraëderș und fället von den 4 andern Puncten Perpendikel auf die Oberflächen des Tetraëders, so mögen sich dieselben verhalten:

wie $a_{1}^{\prime}: a_{2}^{\prime}: a_{3}^{\prime}: a_{4}^{\prime}$ für den ersten Punct, wie $a_{1}^{\prime \prime}: a_{2}^{\prime \prime}: a_{3}^{\prime \prime}: a_{4}^{\prime \prime}$ für den zweiten Punct, wie $a_{1}^{3}: a_{2}^{3}: a_{3}^{3}: a_{4}^{3}$ für den dritten Punct, wie $a_{1}^{4}: a_{2}^{4}: a_{3}^{4}: a_{4}^{4}$ für den vierten Punct. 
6. Hesse, über Verwandlungen der Functionen zweiten Grades.

Bestimmt man dann 4 neue Puncte, deren senkrechte Abstände von den Seitenflächen des Tetraëders sich

wie $a_{1}^{\prime}: a_{1}^{\prime \prime}: a_{1}^{3}: a_{1}^{4}$ für den ersten Punct, wie $a_{2}^{\prime}: \boldsymbol{a}_{2}^{\prime \prime}: \boldsymbol{a}_{2}^{3}: \boldsymbol{a}_{2}^{4}$ für den zweiten Punct, wie $a_{3}^{\prime}: a_{3}^{\prime \prime}: a_{3}^{3}: a_{3}^{4}$ für den dritten Punct, wie $a_{4}^{\prime}: a_{4}^{\prime \prime}: a_{4}^{3}: a_{4}^{4}$ für den'vierten Punct

verhalten, so schneiden sich in diesen 4 Puncten und in den vier Ecken des Tetraëders auch drei Oberflächen zweiter Ordnung.

Der zweite Lehrsatz giebt folgenden geometrischen Satz, unter den Voraussetzungen des eben genannten Satzes:

Die 4 Puncte, deren senkrechte Abstände von den Seitenflächen des Tetraëders sich

wie $\frac{1}{a_{1}^{\prime}}: \frac{1}{a_{2}^{\prime}}: \frac{1}{a_{3}^{\prime}}: \frac{1}{a_{4}^{\prime}}$ für den ersten Punct, wie $\frac{1}{a_{1}^{\prime \prime}}: \frac{1}{a_{2}^{\prime \prime}}: \frac{1}{a_{3}^{\prime \prime}}: \frac{1}{a_{4}^{\prime \prime}}$ für den zweiten Punct, wie $\frac{1}{a_{1}^{3}}: \frac{1}{a_{2}^{3}}: \frac{1}{a_{3}^{3}}: \frac{1}{a_{4}^{3}}$ für den dritten Punct, wie $\frac{1}{a_{1}^{4}}: \frac{1}{a_{2}^{4}}: \frac{1}{a_{3}^{4}}: \frac{1}{a_{4}^{4}}$ für den vierten Punct

verhalten, und die vier Ecken des Tetraëders, sind die Schnittpuncte von drei Oberflächen zweiter Ordnung.

Auf den dritten Lehrsatz werde ich Gelegenheit haben bei meinen über die Doppeltangenten der Curven vierter Ordnung angestellten Untersuchungen zurückzukommen.

Königsberg im April 1851. 